\title{
Experimental and CFD modelling of a Progressive Cavity Pump using overset unstructured mesh
}

\author{
Deisy Becerra*1, Miguel Asuaje ${ }^{2,3,}$, Alexander Zambrano ${ }^{4}$ and Nicolás Ratkovich ${ }^{1}$ \\ ${ }^{1}$ Grupo de Diseño de Productos y Procesos, Department of Chemical and Food Engineering, \\ School of Engineering, Universidad de Los Andes, Bogotá 111711, Colombia \\ ${ }^{2}$ Universidad Simón Bolivar, Department of Energy Conversion and Transport \\ Cra $59 \mathrm{~N}^{\circ} 59-65$, ZP: 1086, Caracas - Venezuela \\ ${ }^{3}$ Frontera Energy \\ C1l 100 \#9-25, Bogotá, Colombia \\ ${ }^{3}$ Frontera Energy \\ ${ }^{4}$ Slacol BCP Group \\ Autopista Medellín Km 10.5 Costado Norte al Interior del Camellon Hercules Km 0+440 Mts. Tenjo, Cundinamarca
}

\begin{abstract}
A Progressive Cavity Pump (PCP) is widely used in industry as an artificial lift method because of its high efficiency during the pumping of high viscosity fluids and two-phase liquid-gas flow slurries. However, modelling PCP through Computational Fluid Dynamics (CFD) is quite complicated since it requires a meshing algorithm and is computationally expensive. Therefore, this study's main objective is to develop a CFD model capable of predicting a progressive cavity pump's behavior by implementing the Overset Mesh, which includes the relative motion between the rotor and the stator. Overset meshes are used to discretize a computational domain with several different meshes that arbitrarily overlap each other. They are most useful because the rotor geometry can be enclosed in a fluid (background) region and set to different positions. The PCP analyzed in this study is a GRP 4.0-4000 208 TSL 1-2 mono-lobe, which contains an API J55 stainless steel rotor and stator handling four Newtonian fluids (water, oil API 11, oil API 22, and oil API 31) at three rotational speeds (100 rpm, $150 \mathrm{rpm}$, and $200 \mathrm{rpm})$. The experimental data presented in this paper was collected in the PCP experimental facility of the SLACOL BCP Group (Tenjo, Colombia). All the measurements were made using the CILA2S controller for artificial lifting in the underground and on the surface to determine the operational curves of flowrate, volumetric efficiency, torque, and power consumed. The CFD model implementation was developed on Star- CCM+ version 15.02-R8 of 2020 for laminar and turbulent regimens. The results obtained through this study show that it is unnecessary to program a structured mesh to capture a progressive cavity pump's performance since the operational parameters evaluated to have an accuracy of $10 \%$ concerning the experimental data.Similarly, capturing the viscous effect near the wall and the transversal y horizontal slip inside the cavities is possible. The flow rate obtained for higher viscosity oils is more significant for the same pressure differences for water with average volumetric efficiencies of $85 \%$. Finally, the pressure increase per stage is homogeneous along the pump's entire length for all fluids evaluated
\end{abstract}

\section{Introduction}

A progressive Cavity Pump (PCP) is a positive displacement pump devised by Moineau in 1930 [1] used as an effective artificial lift method, especially in heavy oil production due to its numerous technical advantages, such as low power consumption and low initial investment [2]-[3]. These types of pumps are capable of handling a broad spectrum of fluids. The range of applications extends from pumping water out of deep wells to transport highly viscous fluids intermixed with solid particles or even liquid-gas flow [4]. The most common PCP is a 1-2 mono-lobe and is composed of a mobile metal rotor and a stationary section (stator) internally coated with elastomer in the form of a double helix. The rotor centreline's hypocycloid trajectory around the stator centreline generates a nutation rotation movement opposite the rotor rotation direction. Because the number of rotor and stator lobes differs by one, a fluid-filled enclosed cavity is formed between a rotor and a stator. During the rotor revolution, the rotor centreline's nutation movement to the stator centreline causes the enclosed cavity to be displaced along the pump axis from inlet to outlet. Hence, when the pump is driven, the movement between the rotor and the stator creates progressive cavities where the fluid moves. As the rotor turns, these cavities move or progress in a combined translation and rotation movement that manifests itself in a helical movement of the cavities from the suction to the pump [5]-[7].

Since its invention in 1930, numerous experimental studies have been developed under single and two-phase flow conditions. Belcher (1991) [8] presented a single-lobe one stage pump in his doctoral thesis. He carried out experimental tests operating with water as working fluid under three rotational speeds, a wide range of pressures, and temperature. He determined that the pressure inside the cavity depends on the rotor's position inside the stator. Later, G. Vetter and W. Wirth (1995) [4] report the influence of rotor-stator interference on different operating parameters of a PCP. Olivet (2002) [9] and Gamboa (2002)[9][10] conducted experiments to understand the effect of viscosity and rotation speed for three different types of crude oil and water, showing that the most viscous fluids can cover a broader range of pressure differences and the volumetric efficiency obtained for oils is more significant than water. Also, [10] in his 
study, carried out the first CFD modelling in two dimensions of a progressive cavity pump, taking into account the infinite approximation of parallel plates and the untwisted model, assuming a Newtonian, incompressible, and isothermal fluid for the approximation of the pressure and velocity profile of the pump, to perform an analysis of the effect of negative interference in a metal-metal progressive cavity pump.

Also, Andrade (2011) [11] made an asymptotic model that describes the single-phase flow within a PCP using the lubrication theory, where the local separation corresponds to the distance between two nonparallel surfaces, which represent the distance between the rotor and stator. The first 3D transient model for single-phase flow was developed by Paladino (2011) [12] based on the previous work of Lima (2009) [13] and Pessoa (2009)[14]. The research work describes the construction of a mesh using FORTRAN, which includes the relative movement between the stator and the rotor through a structured moving mesh for a metal-to-metal PCP. The governing equations are solved using an element-based on the Finite Volume Method. This algorithm is available to predict the operational curves as the pump's volumetric efficiency and the viscous losses, in addition to the pressure and velocity profiles within the device. The numerical solution obtained with commercial CFD software is hugely complicated and computationally expensive. The CFD results were compared with the available experimental data of Gamboa (2002) [9], with a maximum discrepancy of approximately $20 \%$.

Another recent work was presented by Chen et al. (2013) [15] calculated the transversal and longitudinal slip also considering FSI for the stator deformation caused by pressure variations along with the pump and subtracted from the displaced flow rate even-thickness PCPs and study the influence of different material and structure parameters on the internal slip of PCP. Moreover, Nguyen et al. (2014) [16] used a vector approach and the hypocycloid theory to determine the theoretical pump factor for any given PCP as a function of the eccentricity, the number of lobes, semicircle diameter, and pitch length. Also, Nguyen (2016) [17] developed a model for predicting and optimize a multi-lobe PCP performance from the use of two models available in the literature, called an analytical model to predict the theoretical pump performance and the slippage model handling single-phase flow (liquids 0 to $450 \mathrm{cp}$ ). The results of this study demonstrate the influence of pump clearance on global performance. If the clearance is smaller than a specific value, then the effects of differential pressure and liquid viscosity are minimal.

On the other hand, Mrinal et al. (2016) [18] presented the first 3D model with the Dynamic Mesh model using ANSYS Fluent to predict the flow variables at several rotational velocities. This model was used for the rotor hypocycloid movement, and it was defined as a function for the algorithm user written in $\mathrm{C}$ language. The mesh deformation was developed using springbased smoothing and local re-meshing technique models. AlSafran (2017)[19] created a set of four theoretical, operational, fluid properties and pump geometry, dimensionless groups that govern the fluid behavior inside a PCP. Those numbers are the Euler number, inverse Reynolds number, specific capacity number, and Knudsen number derived from the Navier Stokes equation and specific boundary conditions.

However, all the above studies are computationally demanding and complicated to implement since they require the programming of a structured moving grid. Therefore, the purpose of this paper is to present a simple alternative for modeling a progressing cavity pump using an unstructured chimeric mesh, called Overset Mesh, for pumping single-phase fluids of water and crude oils of different viscosity. This study is valuable since it is the first to apply two overlapping meshes for modeling a PCP, obtaining errors below 5\% concerning the experimental data. Likewise, the CFD model can approximate the transverse and longitudinal slip inside the pump, which previous studies have not reported.

\section{Methodology}

The present section is divided into two main parts. The first part discusses the experimental facility and procedure out for this work. The second part refers to the pump's CFD modeling, which includes a detailed description of the new unstructured moving mesh method, called overset mesh, the boundary conditions, and selected physical models. The fluids used were chosen to represent the mixtures' rheological characteristics commonly found in mature, heavy crude oil wells. In this case, only the study of the pump's performance under single-phase flow conditions is assumed.

\section{Experimental methodology}

The experimental data presented in this paper was collected in the PCP experimental facility of SLACOL BCP Group (Tenjo, Colombia), shown in Figure 1. The PCP analyzed in this study is a GRP 4.0-4000 208 TSL 1-2 monolobe, which contains an API J-55 stain steel elastomer and rotor. All the measurements were made using the CILA2S controller for artificial lifting in the underground and on the surface. It is the standard solution of SLACOL for stand-alone assembly in a skid in operation and optimization of a well. CILA2S is provided with all the necessary equipment for the operation and production of oil wells. Its architecture is based on variable speed drive (VSD), process control processors according to its advanced capacity of optimization of a production cluster. The tests were performed at different rotational speeds $(100,150$, and $200 \mathrm{rpm})$ and discharge pressures. Allocated along this closed loop is a series of instruments detailed in 


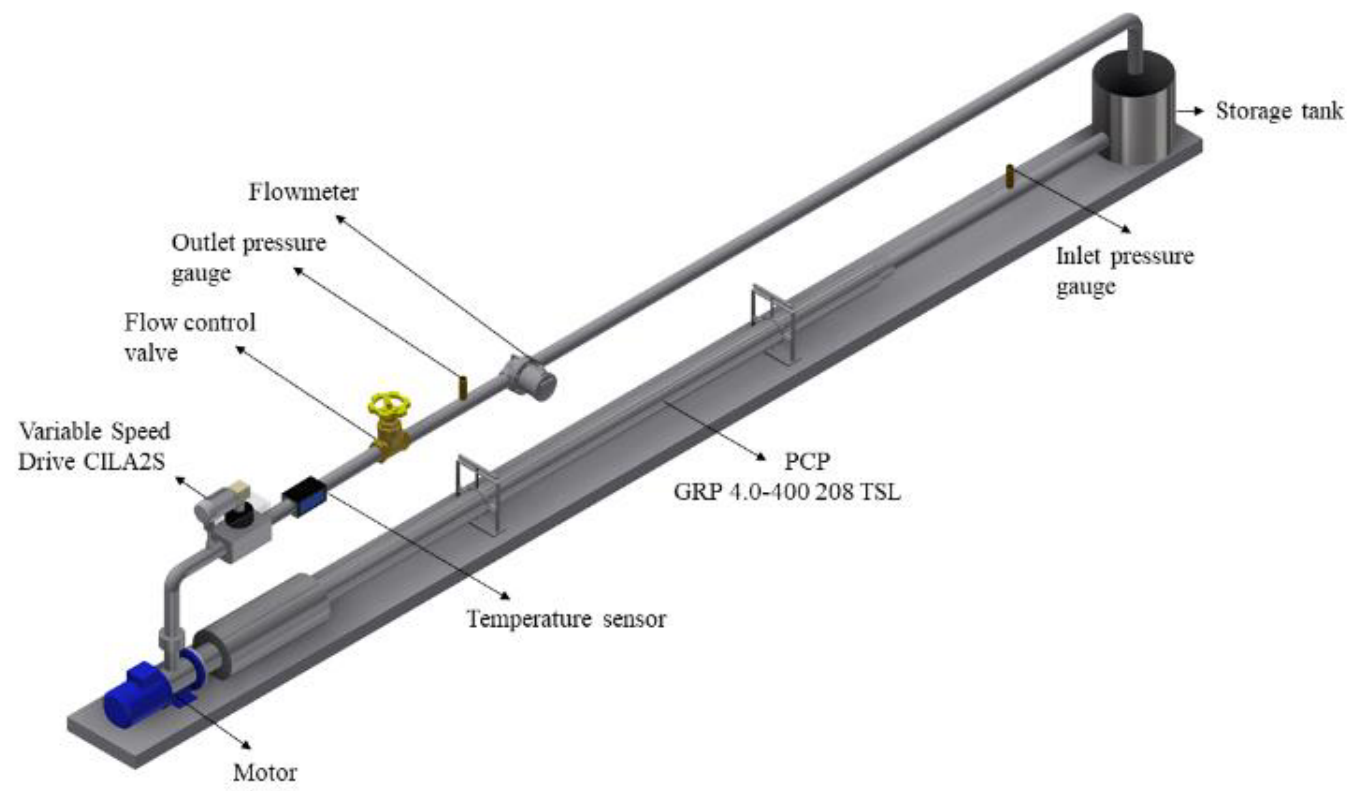

Figure 1. SLACOL PCP experimental setup

The experimental unit comprises a tank where the PCP is installed and connected to a 5-1/2" stain steel pipeline. This pipe recirculates the pumped fluid back to the tank. The setup is designed to handle a maximum differential pressure of 1470 psi. In this case, the experimental procedure consisted of manipulating the PCP's flowrate through a flow control valve installed at the outlet section of the pump loop, as shown in Figure 1, for each rotational speed evaluated. The flowrate handled was measured using a magnetic flowmeter Sitrans FM MAGFLO MAG6000 suitable for measuring the flow rate of almost all liquids, pastes and slurries, until the required operating pressure is reached. Once a steady-state operation was attained, pressure rise measurements were taken with a wide range of digital pressure sensor Danfoss model MBS 3000. For each flowrate considered, the electrical and operational tests and measurements were performed using CILA2S PCP (power and torque) connected to the motor shaft. This same procedure was performed for each rotation speed evaluated in all fluids until an operation curve was built with at least five pressure and volumetric flow points.

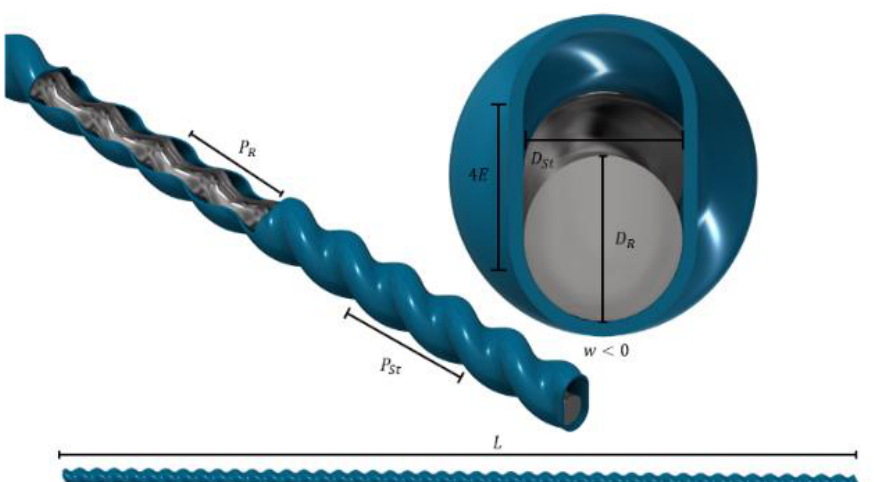

Figure 2. PCP 3D CAD model

Table 1. Characteristics of the main test measuring instruments

\begin{tabular}{|c|c|c|c|}
\hline Equipment & Brand & Model & Details \\
\hline Magnetic flowmeter & SIEMENS & $\begin{array}{c}\text { Sitrans F M MAGFLO } \\
\text { MAG6000 }\end{array}$ & $\begin{array}{l}\text { Accuracy class: } \pm 0,2 \% \pm 1 \mathrm{~mm} / \mathrm{s} \\
\text { Temperature range: }-25 \text { to }+60{ }^{\circ} \mathrm{C} \\
\text { Voltage: } 100 \text { to } 230 \mathrm{VAC} \text { (of } 47 \mathrm{a} 63 \mathrm{~Hz}) \\
\text { Level of protection: IP } 67 / \mathrm{NEMA} 4 \mathrm{X} \text { according to } \\
\text { IEC } 60529 \text { and DIN } 40050(1 \mathrm{mH} 2 \mathrm{O} 30 \mathrm{~min})\end{array}$ \\
\hline Pressure sensor & Danfoss & MBS 3000 & $\begin{array}{l}\text { Measure range: } 0 \text { - } 9000 \text { psi } \\
\text { Accuracy class: } \pm 1 \% \\
\text { Temperature range: }-40 \text { to }+85^{\circ} \mathrm{C} \\
\text { Voltage: } 10 \text { to } 30 \mathrm{~V} \\
\text { Level of protection: IP65 - IP67 }\end{array}$ \\
\hline
\end{tabular}




\begin{tabular}{c|l|l|l}
\hline Equipment & Brand & Model & \multicolumn{1}{c}{ Details } \\
\hline & & & Measure range: 0 $-500^{\circ} \mathrm{C}$ \\
\hline Temperature sensor & Pixsys & PT100B & $\begin{array}{l}\text { Measure range: 0 to } 500{ }^{\circ} \mathrm{C} \\
\text { Accuracy class: } \pm 30{ }^{\circ} \mathrm{C} \\
\text { Material: AISI 304 steel }\end{array}$ \\
\hline
\end{tabular}

The fluid's properties analyzed in this thesis and used as input in the CFD simulation studied were measured in the SLACOL laboratory and are presented in Table 2. According to the experimental data that the company has in different locations such as Teak 11, Chichimene SW-08, and Remanso BSW, three conventional crude oils with different viscosities and densities were selected 95 wells.

Table 2. Tested fluid properties.

\begin{tabular}{l|c|c|c}
\hline \multicolumn{1}{c|}{ Fluid } & $\begin{array}{c}\text { Viscosity } \\
{[\mathrm{Pa} . \mathrm{s}]}\end{array}$ & $\begin{array}{c}\text { Density } \\
{\left[\mathrm{kg} / \mathrm{m}^{3}\right]}\end{array}$ & $\begin{array}{c}\text { Surface } \\
\text { tension } \\
{[\mathrm{mN} / \mathrm{m}]}\end{array}$ \\
\hline Water & 0.001 & 997 & \\
Crude Oil API 12.8 & 3.314 & 992.4 & 32.5 \\
Crude Oil API 22.6 & 0.164 & 917.2 & 28.34 \\
Crude Oil API 31.54 & 0.01311 & 866.626 & 26 \\
\hline
\end{tabular}

\section{Numerical methodology}

The CFD modeling was carried out using commercial code STAR-CCM+, version 15.02-R8 of 2020.

\section{Geometry Modelling}

The geometry model was developed in detail using the software Autodesk Inventor 2021. The computational domain set consists of the rotor and stator, with the total number of pitches. The main geometrical dimensions for PCP are presented in Table 3. All dimensions were taken from the pump's construction drawing. 3D CAD is illustrated in Figure 2.

Table 3. PCP geometry specifications

\begin{tabular}{l|c|l}
\hline & Parameter & Value \\
\hline $\begin{array}{l}\text { Rotor } \\
\text { Minor Rotor diameter [mm] }\end{array}$ & $D_{R}$ & 42 \\
Rotor pitch [mm] & $P_{R}$ & 100 \\
Stator & & \\
Minor Stator diameter [mm] & $D_{S t}$ & 42.37 \\
Stator pitch [mm] & $P_{S t}$ & 200 \\
$P C P$ & & \\
Clearance [mm] & $w$ & 0.185 \\
Eccentricity [mm] & $E$ & 8 \\
Number of stator pitches & $n_{P}$ & 27 \\
Total length [m] & $\mathrm{L}$ & 5.4 \\
Nominal flowrate [bbl/day] at & $Q_{\text {nom }}$ & 207.6 \\
100 rpm & &
\end{tabular}

However, it is essential to consider that to reduce the simulation time, the pump's length was reduced to $1.2 \mathrm{~m}, 6$ stator pitches, and the experimental data were taken in this distance.

\section{Simulation Procedure}

Figure 3 represents the computational domain of the progressive cavity pump (PCP), whose geometry was divided for the CFD model. Four types of regions and boundary conditions were considered: i) the inlet section, which was modeled as a Stagnation Inlet; ii) the outlet section, which was taken as Pressure Outlet with a working pressure changed according to the operational difference pressure studies (0 to $300 \mathrm{psi}$ ); iii) the stator wall region, that was modeled as no-slip wall and finally iv) the overset region which was modeled by generating a $6 \mathrm{~mm}$ shell on the surface of the rotor, to generate the active cells of the moving mesh (see section 80.). As for the initial conditions, velocity was set to $0 \mathrm{~m} / \mathrm{s}$ in all directions, and the pressure was taken as the atmospheric pressure of Tenjo, Cundinamarca $(101700 \mathrm{~Pa})$, where the experiments were carried out.

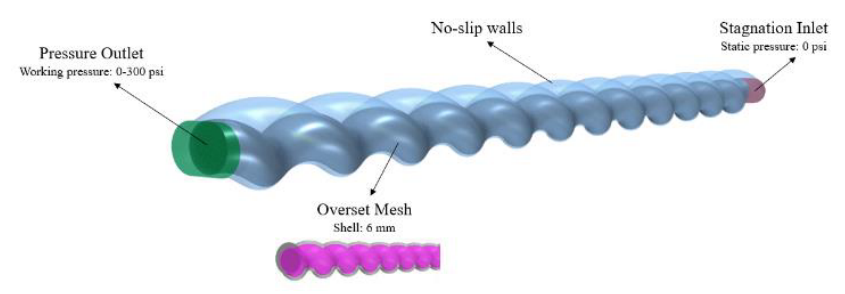

Figure 3. PCP's boundary conditions

For pump rotation, it was considered a Rigid Body Motion (RBM) model since it is the most accurate in STAR-CCM+ to simulate moving parts. The RBM can capture transient flow effects such as rotor transversal movement [20]- [21] (see pump kinematic specification in section 0). Moreover, a time step of $0.001 \mathrm{~s}$ was considered to achieve $1^{\circ}$ of rotation with 200 inner iterations per time-step. The simulations were run up to $1 \mathrm{~s}$. which roughly corresponds to 3 revolutions of the rotor, to obtain a representative steady-state solution. The Courant number was also reported for all simulation time and obtained a range of 0.005-0.5 (See Figure 5). The geometrical o theoretical transport flow can be calculated considering the cavity volume $V$ and the number of rotations per minute of the pump by Eq. (1.

$$
Q_{t h}=4 E \cdot D_{R} \cdot P_{s} \cdot N
$$


Nevertheless, since in this study a PCP with negative interference was used, it is necessary to modify the previous equation considering the clearance parameter, obtaining

$$
\begin{gathered}
Q_{t h}=\left[4 E \cdot D_{R}-8 E \cdot w-\pi \cdot\left(D_{R} \cdot w+w^{2}\right)\right] \\
\cdot\left(P_{S} \cdot N\right)
\end{gathered}
$$

Finally, the actual flowrate $Q$ is determined considering a leak rate or internal slip flow, which is:

$$
Q=Q_{t h}-S
$$

The internal slip flow $S$ is the phenomenon that defines the PCP performance, due to it is a function of the pump's geometry specifications, the fluid properties, and operational variables. It is described as a strong transitory and non-fully developed flow through clearances because it changes according to the rotor position. Therefore, it is possible to analyze it through two approaches: longitudinal slip and cross-sectional slip [22], presented in Figure 4.

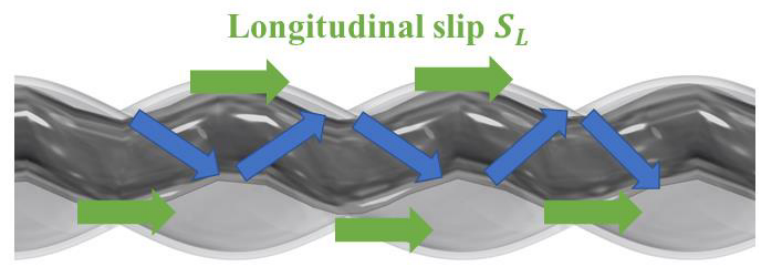

Cross-sectional slip $S_{c}$

Figure 4. PCP internal slip

Hence, based on the formulation given above, an expression for the volumetric efficiency can be written, as shown in Eq. (4.

$$
\eta_{v}=\frac{Q}{Q_{t h}}
$$

On the other hand, the power consumption is determined by the effective hydraulic power $P_{h}$, the load $P_{S}$ of the internal slip, the losses related to friction power $P_{f}$ and the mechanical power $P_{m}$.

$$
P=P_{h t h}+P_{f}+P_{m}=P_{h}+P_{s}+P_{f}+P_{m}
$$

The hydraulic power is defined by the pump geometry, the rotational speed, and the pressure difference. Hence, the expression is written as Eq. (6.

$$
P_{h}=Q \cdot \Delta P
$$

The power loss associated with the pump's internal slip flow is calculated in the same way as the hydraulic power.

$$
P_{S}=S \cdot \Delta P
$$

The losses understood as friction are determined through the coefficient of friction between the rotor/stator. Similarly, it is affected by the properties of the fluid, precisely its viscosity.

$$
P_{f}=P(\mu, \text { lubrication })
$$

Finally, the mechanical power can be described using the rotor torque and the operational rotation speed.

$$
P_{m_{n e t}}=\tau_{z} \cdot \omega
$$

Hence, the mechanical and isentropic hydraulic efficiency is defined as:

$$
\begin{gathered}
\eta_{h}=\frac{P_{h}}{P_{m_{\text {net }}}}=\frac{\Delta P Q}{\tau_{z} \omega} \\
\eta_{m}=\frac{P_{m_{\text {net }}}}{P_{m_{\text {drive }}}}
\end{gathered}
$$

The volumetric flow was calculated using an integral report of velocity on the outlet section. The torque was also estimated to employ a momentum report in $-Z$ direction, and the other operational variables were calculated using scalars field functions. These were reported on surface average monitors until a stable behavior is obtained (approximately from $0.001 \mathrm{~s}$ to $1 \mathrm{~s}$ ).

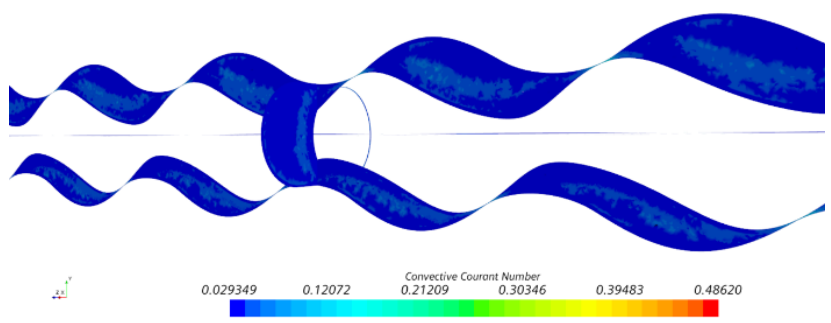

Figure 5. Water Convective Courant Number at 100 psi

For the multiphase simulations, the initial volume fractions were changed according to the operational point studied, and the primary phase was defined considering the phase of greater quantity as the continuous one. The interaction properties as the interfacial and surface tension were specified in the multiphase interaction model.

\section{Physical Model Specification}

The physical model used in each simulation were selected following different criteria. To know whether a turbulent approximation should be used, the Reynolds number $(R e)$. The results obtained are shown in Table 4. All simulations where the Reynolds number was below 2100 were considered laminar. With this criterion, the only monophasic simulations modeled as turbulent were dealing with water as the pumping fluid. For these, the mass and momentum equations were solved using a U-RANS approach in which the RST was solved using the Boussinesq approximation. The turbulent viscosity for the calculation of the RST was obtained by using a twoequation eddy-viscosity turbulence model, specifically the Realizable $k-\varepsilon$ turbulence model coupled with a Twolayer all $\mathrm{y}+$ wall treatment. This approach was selected given that zero, and one-equation models do not entirely describe turbulence length scales on their own, while two- 
equation models are complete in the sense that they only require initial and boundary conditions for it to be used. Also, the $k-\varepsilon$ turbulence model was chosen over other types of two-equation models given that it is robust and acceptably computationally accurate enough while having a low computational cost. The Realizable variant of the $k-$ $\varepsilon$ turbulence model variant was used given that it has demonstrated a superior capability to capture the mean flow of complex structures and rotatory flows concerning standard $k-\varepsilon$. Given the problems of the turbulence model selected to model turbulence in regions near the wall, a coupling with a two-layer all $\mathrm{y}+$ wall treatment was used.

Table 4. Reynolds number calculated for each monophasic simulation at different rotor rotational speed

\begin{tabular}{c|ccc}
\hline \multirow{2}{*}{ Fluid } & \multicolumn{3}{|c}{ Reynolds Number } \\
\cline { 2 - 4 } & $\mathbf{1 0 0} \mathbf{~ r p m}$ & $\mathbf{1 5 0} \mathbf{~ r p m}$ & $\mathbf{2 0 0} \mathbf{~ r p m}$ \\
\hline Water & 20700 & 31100 & 41500 \\
API 12.8 & 2.11 & 3.16 & 4.21 \\
API 22.6 & 110 & 166.00 & 221 \\
API 31.54 & 916 & 1370 & 1830 \\
\hline
\end{tabular}

On the other hand, given the monophasic oil simulations, given the flow's laminar nature, the standard equations of continuity and momentum were solved. It is essential to clarify that all simulated fluids were assumed to be incompressible and that the situation modeled was consider isothermal, which is why thermal energy transport equations were not considered. To numerically solve the transport equations, a finite volume element approach was used. A second-order discretization scheme for diffusive and convective terms was used while solving the transient part of the equations an implicit first-order Euler scheme was used. It is unconditionally stable, requiring less computational effort to calculate a time step than a secondorder or higher-order scheme. A constant time step of 1.6 $\mathrm{ms}$ was used for $100 \mathrm{rpm}, 150 \mathrm{rpm}$, and $200 \mathrm{rpm}$ respectively, which represents approximately $1.5^{\circ}$ degrees of rotation per time step, which is sufficiently low for the overset mesh solver to do an accurate whole cutting per step as well as to maintain the convective CFL number below 1 .

For the overset mesh configuration, an alternate hole cutting approach was selected even though it is computationally more expensive than other hole cutting approaches, given that this method is robust for cases where there are small gaps in the geometry. This is the case of the PCP, where small gaps between the rotor and stator are present and play an essential role in the pump dynamics directly affecting the slip flow. Also, a prims layer shrinkage option was selected to guarantee that at least 4 layers of the cell are between the overset and the background region in the small gap zones; considering this, 10 adaptative prims layers for each mesh were configured with a stretch factor of 100 .

To guarantee that physical frontiers are correctly preserved in the hole cutting process (such as the stator walls in the PCP), the close proximity option was selected to prevent mesh intersection failure when the overset region overlaps the background's physical boundaries region. Finally, a linear interpolation scheme was used given that it is more accurate and does not allow interpolation elements to overlap, despite being more computationally expensive. Likewise, to reduce interpolation mass unbalance, a mass tracking conservation option was used.

\section{Pump kinematics}

The motion of a PCP is governed by a hypocycloid curve [18]. This curve is described by the rotation of a small circle of radius $r$ which rolls within a bigger circle of radius $R$, as it is shown in Figure $6 a$.

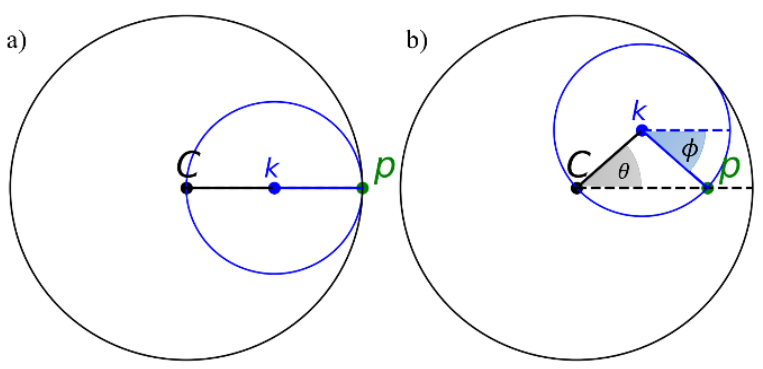

Figure 6: Representation of the generation of the hypocycloid curve where a) is the initial situation $(\boldsymbol{t}=\mathbf{0})$ and $\mathbf{b})$ is a situation at a posterior time $\boldsymbol{t} \neq \mathbf{0}$

Consider the position of the center of the big and the small circle $C$ and $k$, respectively. The position of the point $k$ is going to be given by the vector

$$
k=\left[\begin{array}{c}
C_{x}+(R-r) \cos \theta \\
C_{y}+(R-r) \sin \theta
\end{array}\right]
$$

Where $\theta$ is the angular position of the $k$ in the circumference of radio $(R-r)$. Now, consider a point $P$ which is at angle $\phi$ (which is counted clockwise), within the small circumference of radius $r$, its position concerning point $\mathrm{C}$ is going to be given by 


$$
P=\left[\begin{array}{l}
k_{x}+r \cos \phi \\
k_{y}-r \sin \phi
\end{array}\right]=\left[\begin{array}{l}
C_{x}+(R-r) \cos \theta+r \operatorname{co} \\
C_{y}+(R-r) \sin \theta-r \sin
\end{array}\right.
$$

Consider that at an initial time $t=0$, the small circle and the point $P$ are in the situation showed in Figure $6 \mathrm{a}$ and that in a further moment $t \neq 0$ the small circle have rolled a certain angle $\theta$ with respect to its original position. In this case, the displacement made by the small circle must be equal to the displacement of point $P$, which means that the length of the arc of the movement of the small circle (the arc between it is the two black lines in Figure 6b) and the length of the arc of the movement of point $P$ (the arc between the solid blue line and the dashed black line in Figure 6b) must be equal

$$
R \theta=r(\theta+\phi)
$$

Replacing this last equality into the position $P$ and defining $v$ as the ratio $\frac{R}{r}$, the following expression is obtained.

$$
P=\left[\begin{array}{c}
C_{x}+r(v-1) \cos \theta+r \cos ((v-1) \theta) \\
C_{y}+r(v-1) \sin \theta-r \sin ((v-1) \theta)
\end{array}\right]
$$

This last expression describes what is known as the parametric equation of a hypocycloid. In this last equation, the form of the curve will be given by the value of $v$, which indicates the number of cusp or sharp corners the trajectory will have. For the case of a mono lobe PCP, the value of $v=$ 2 , which means that the trajectory of point $P$ is going to have two cups and that the relationship between the angle $\theta$ and $\phi$ is going to be $\theta=\phi$ (as given by equation 21). The trajectory of point $P$ is then going to be provided by

$$
P=\left[\begin{array}{c}
C_{x}+r \cos \theta+r \cos (\theta) \\
C_{y}+r \sin \theta-r \sin (\theta)
\end{array}\right]=\left[\begin{array}{c}
C_{x}+2 r \cos \theta \\
C_{y}
\end{array}\right]
$$

Considering that the angular velocity of rotation of the small circle is constant $(\omega)$, the angle in every moment is going to be given by $\theta(t)=\omega t+\theta_{o}$. With this in mind and observing the position of the point $P$ when $v=2$, this point describes a constant oscillatory motion on the $x$ axis. Now considering the point $P$ as the center of the rotor and $r$ as the eccentricity of the stator $(\varepsilon)$, the PCP movement observed in its cross-section will be as the one shown in Figure 7.

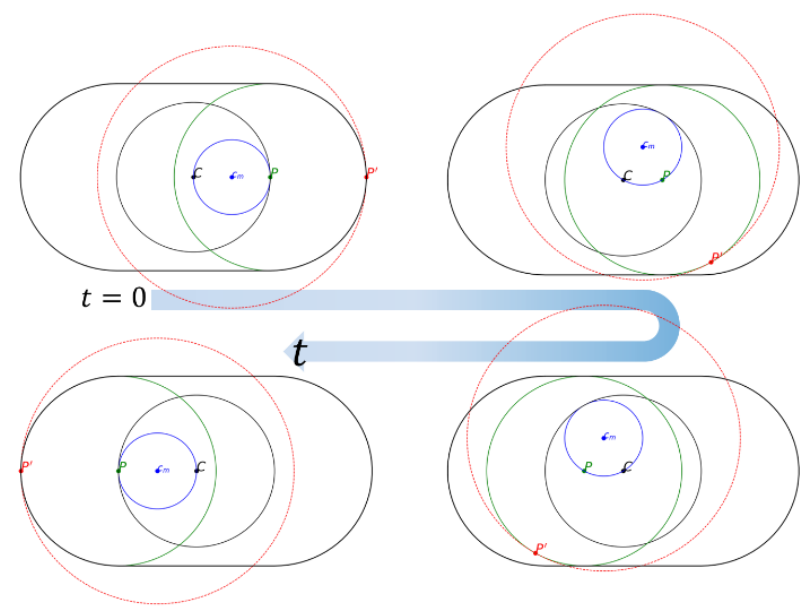

Figure 7. Cross-section view of the rotor movement as a function of time for half a period, where the green circle represents the rotor with center $\boldsymbol{P}$, the black lines represents the stator wall with the center in $\boldsymbol{C}$ and the blue and black circles represent the hypocycloid followed by the rotor and $\boldsymbol{c}_{\boldsymbol{m}}$ represents the center of mass of the rotor. The motion of the rotor is described by a translation and a rotation around its axis $\boldsymbol{P}$

In Figure 7, the movement of the rotor in a horizontal cross-section is characterized by the oscillatory motion of the central point of the rotor $(P)$, and a rotation of the rotor about its center (which can be observed by following the trajectory of the point $P^{\prime}$ along the rotor movement). In that sense, a point $P^{\prime}$, located at the external radius of the rotor will follow the next motion

$$
P^{\prime}=\left[\begin{array}{c}
2 e \cos \omega t+R_{r} \cos \omega t \\
-R_{r} \sin \omega t
\end{array}\right]
$$

Now given that the motion of the PCP is fully understood as a simultaneous rotation ad translation of the rotor in the stator, this movement is going to be implemented within STAR-CCM+. To do this, it is essential to consider the whole rotor as one singles piece, which rotates around its center of mass and has a translation given by the hypocycloid curve. As stated before, the translation of the center of mass is going to be provided by

$$
k=c_{m}=\left[\begin{array}{c}
(R-r) \cos \theta \\
(R-r) \sin \theta
\end{array}\right]=\left[\begin{array}{l}
e \cos \omega t \\
e \sin \omega t
\end{array}\right]
$$

Given that the program Star-CCM+ requires the translational velocity of the center of mass, this is going to be given by 


$$
v_{c_{m}}=\left[\begin{array}{c}
-e \omega \sin \omega t \\
e \omega \cos \omega t
\end{array}\right]
$$

On the other hand, the rotation about the center of mass of the rotor is only specified in Star-CCM+ by its rotation rate and which is the same as the one used in the translational velocity of the center of mass $(\omega)$, but with a negative sign, given that the angle $\phi$ which is the angle of rotation around the center of mass is considered positive in the clockwise direction. With this information, the rotor's motion is fully specified in Star-CCM+, and it can be solved using the Rigid Body Motion algorithm.

\section{Overset Mesh configuration}

The workflow followed in STAR-CCM+, as suggested in its documentation, is summarized in 5 simple steps:

Individual regions are defined: A typical overset simulation has a background region enclosing the entire solution domain, the fluid, and one overset region surrounding a body such as the PCP's rotor. The outer surface of the overset region is defined as a boundary [23]. Hence, to limit the overset region, a shell was created around the rotor with a thickness of $6 \mathrm{~mm}$. Subsequently, the intersection between these two bodies was made to obtain the rotor's internal volume.

The regions have meshed separately and independently: The meshing process was carried out in each region. For the base mesh generation, two types of elements were considered: polyhedrons with a base size of $5.5 \mathrm{~mm}$ and prisms layers. The remaining volume was built with polyhedrons for both meshes. The polyhedral mesh model provides a more numerically stable, precise, and less diffusive mesh than an equivalent tetrahedral mesh [24]. These advantages are obtained because polyhedrals offer a more significant number of cell faces, resulting in two characteristics: i) a more substantial number of immediate neighboring cells, which increases the accuracy of the calculations and ii) a greater probability of finding a cell face that is aligned with the direction of flow, which decreases numerically diffusivity [25]. Alignment with the flow direction also provides greater precision in the calculations, especially for convective terms [21]. The figure 10 hows the final 3D base mesh generated.

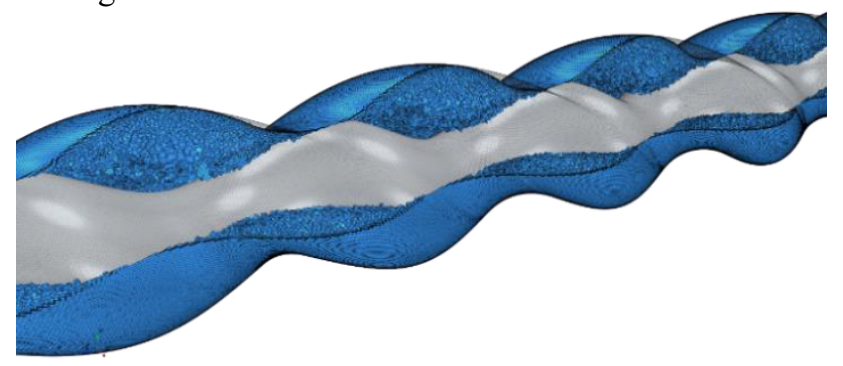

Ten prism layers were in thin and near-wall regions to capture the hydrodynamic boundary layer appropriately. The prism layer total thickness near the wall was set to 0.75 $\mathrm{mm}$ with stretching of $30 \%$. Hence, the first layer was fixed at $0.075 \mathrm{~mm}$. This setting guarantees enough mesh refinement $\left(\mathrm{y}^{+<5)}\right.$ in the first layer to resolve the inner region viscous sublayer directly [26], as seen in Figure 9. Thus, the wall shear stress predictions are improved for lower viscosity fluids (water). Hence, a hybrid all $\mathrm{y}+$ wall treatment model was selected for all two-phase simulations to allow higher mesh refinement flexibility. This model also ensures more accurate calculations where the mesh has an intermediate resolution and lies in the buffer sublayer $(5<y+<30)[27]-[28]$.

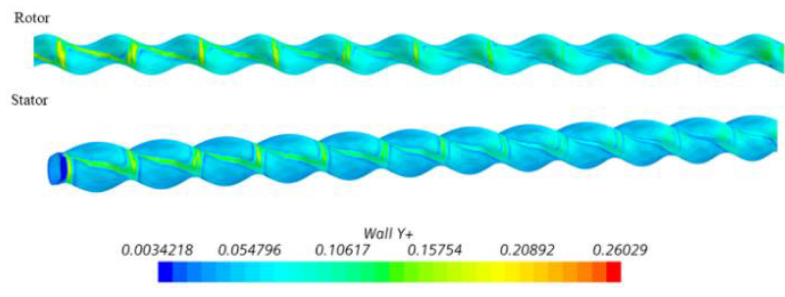

Figure 9. Wall $\mathrm{Y}+$ profile obtained on the rotor and stator for water at 100 psi

The interface between the overset (rotor) and background (fluid) meshes is defined: Moreover, the overset interface was created. The overset boundaries in the overset region define the border of the hole-cutting process, and it is mandatory that 4 to 5 cells layers are always present in the overlapping zone between the background and overset regions to have enough active donor cells that are capable of transmitting information between the rotor mesh and the fluid mesh. In this case, this was ensured by using the 10 prismatic layers. Subsequently, the Region-0 and Region1 were selected. Region-0 identifies the Overset region (rotor) with higher priority. The priority controls the holecutting between Region-0 and Region-1. Also, Region-1 Identifies the Background region.

The Close proximity option was activated because there is a narrow gap between the moving body and the wall boundary. This option allows more active cells in the overset mesh interface and helps prevent mesh intersector failure when the overset region overset region overlaps the background region's physical boundaries. In this case, when the rotor goes close to the fluid wall boundary, the overlapping region can extend over the fluid boundary; those cells remain active as long as the cell centroid is within the background region. The cell is deactivated only when the cell centroid is outside the boundary. Finally, the Prims Layer Shrinkage option was selected to allow the prism layers to contract when moving objects enclosed by wall boundaries approach each other and form a pump's clearance.

Figure 8. PCP 3D mesh 
The overset domain is moving, so the interface's overlapping zone is updated: For each time-step of the simulation, the mesh surrounding the rotor adapts until 3 revolutions are completed. Figure 10 shows the mesh adaptation for different rotor positions.

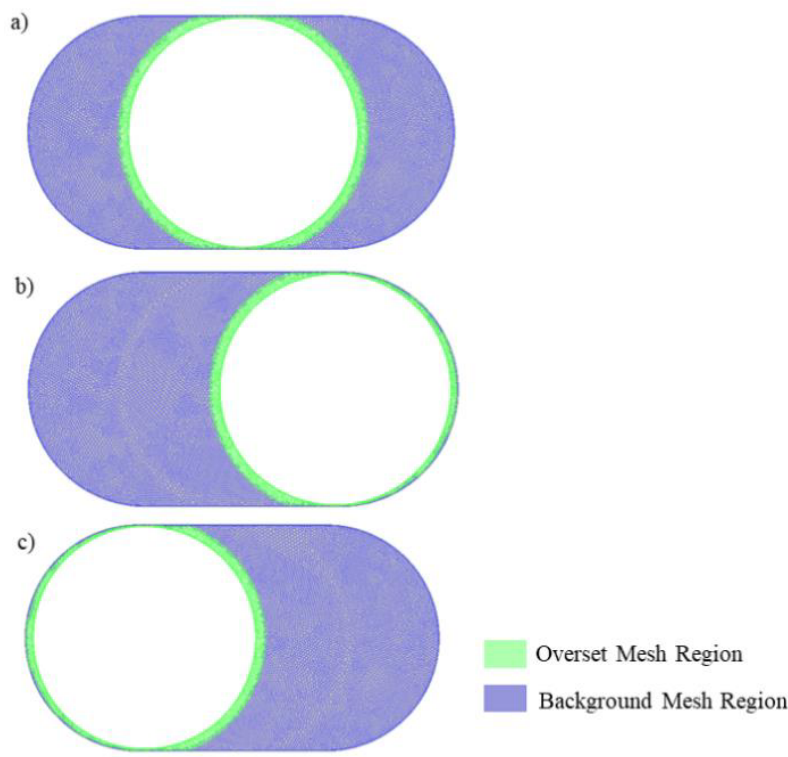

Figure 10. 2D computational mesh for different rotor positions a) initial position $0^{\circ}$, b) $90^{\circ}$, and c) $270^{\circ}$

Data is interpolated and communicated between the regions at the interface: After the hole-cutting process is complete, the cell types of a PCP using Overset Mesh representation are shown below. Figure 11 present the cell topology allows the transmission of information through a linear interpolation scheme.

a)

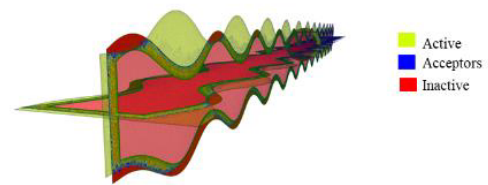

b)

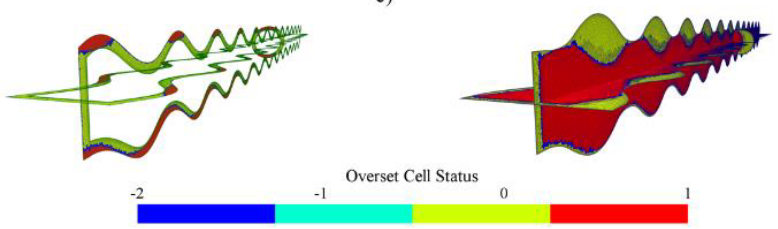

Figure 11. PCP Mesh Topology for a) the final mesh combined from both regions, b) the cell types in the overset region (rotor), and c) the cell types in the background (fluid) region

\section{Mesh Independence Analysis}

To determinate the most favorable number of cells to use for the PCP simulations in terms of computational time required to obtain a complete operational curve and the accuracy of results obtained, a mesh independence test (MIT) and Grid Convergence Index (GCI) method was carried out. In this case, four different mesh sizes were evaluated (Coarse, Base, Fine, and Ultra Fine), hanging the total number of elements from modifications in the base size of each cell, but keeping constant the mesh parameters such as the number of prismatic layers and for the adaptive mesh model the transition width of 4 and a minimum adaptive cell of 1e-6. Firstly, only the results obtained for water were considered for the mesh independence test. Five different simulations were performed for each mesh type, evaluating pressure ranges between 0 and 400 psi at 150 rpm. The flow rate at the pump outlet was calculated and compared with the experimental results, assessing the accuracy of the model through the root-mean-square error using the below equation.

$$
R S M E_{\text {Water }}=\sqrt{\frac{\sum_{i}\left(\frac{Q_{E x p_{i}}-Q_{C F D_{i}}}{Q_{E x p_{i}}}\right)^{2}}{5}}
$$

As shown in Figure 12, as the number of cells increases, the computation time increases, but the result's average accuracy decreases. However, significantly increasing the number of cells implies a higher cost in computational resources. In this MIT, it is possible to evidence that the coarse mesh has twice the base mesh error. Likewise, the fine mesh achieves an increase in accuracy of $0.28 \%$ and the ultrafine mesh of $0.19 \%$, concerning the base mesh. This implies that even if the number of elements is significantly increased, there is no significant error reduction between the experimental and simulated data. Therefore, it is possible to conclude that the CFD model's accuracy becomes independent for meshes with more than 9.5 million elements.

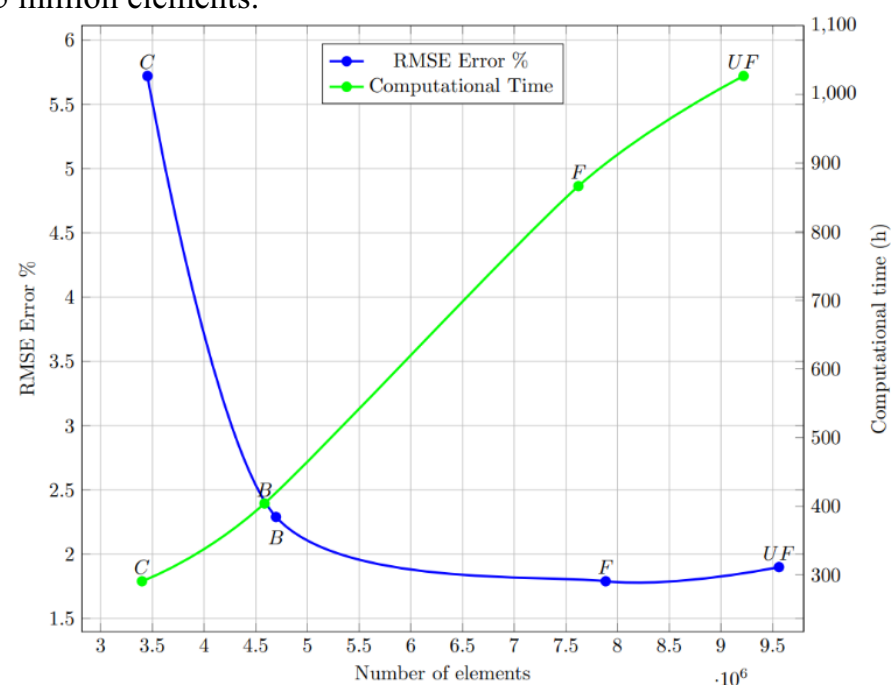

Figure 12. Water Mesh Independence Test 


\section{Results and discussion}

Firstly, to ensure the accuracy of the CFD model implemented in predicting the different operational variables of the PCP.

b)

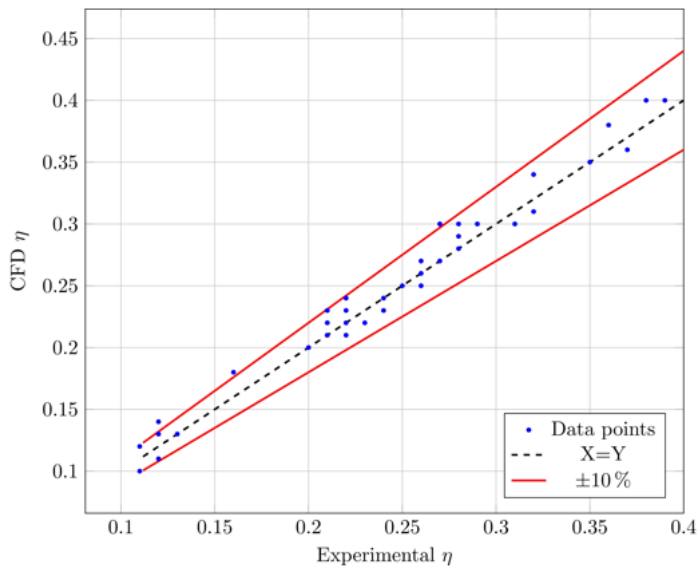

Figure 13 was constructed to visualize the deviation of the total flow rate, mechanical, hydraulic, and volumetric efficiency values regarding the experimental data for singlephase flow for water and the three types of API crude. An excellent agreement can be observed. Almost all the data points are within the $\pm 5 \%$ for the outlet flow rate and $10 \%$ for the PCP total efficiency for the five pressure differences evaluated. This result was expected because the CFD model does not consider the mechanical and friction losses directly related to the PCP's motor and stator material.

a)

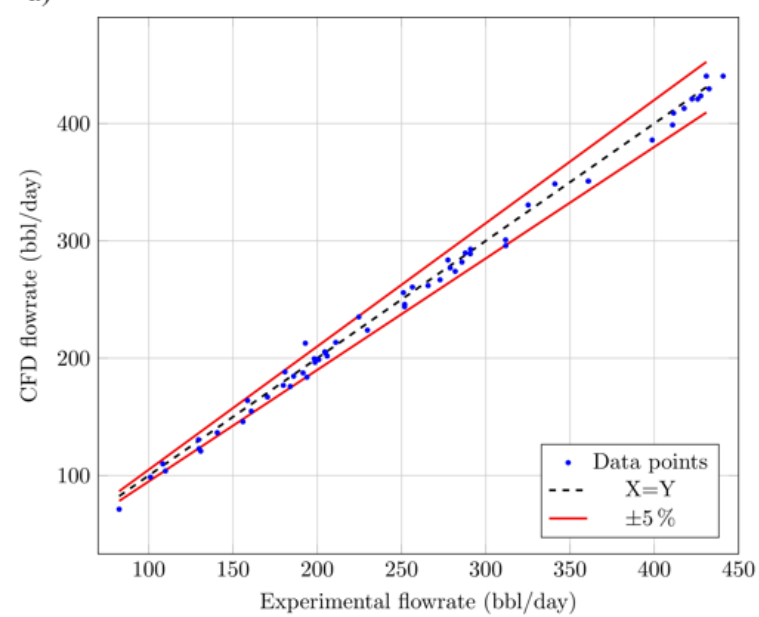

b)

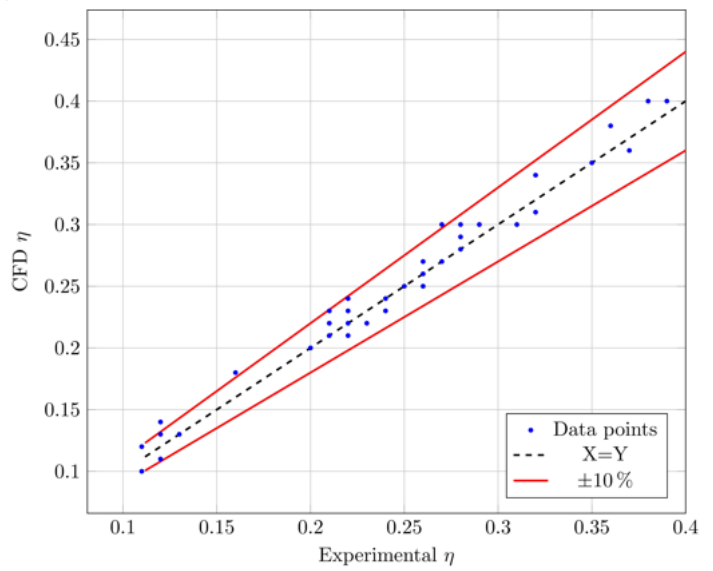

Figure 13. CFD vs. Experimental comparison of a) flowrate and b) Efficiency values for all single-phase fluids tested

Hence, it shows that the CFD model can correctly predict the turbomachine's performance by implementing an automatic and unstructured mesh. Paladino [12] previously mentioned that it was mandatory to build an algorithm with a structured mesh because the angular distortion during the mesh movement could not be modeled by tetrahedral or hexahedral meshes generated automatically by commercial software. However, with today's powerful models, they can fulfill this goal.

\section{PCP performance}

Four fluids were tested: water, oil API 11, oil API 22, and oil API 31 at three rotation speeds of $100 \mathrm{rpm}, 150 \mathrm{rpm}$, and $200 \mathrm{rpm}$.

The volumetric flow rate behavior versus the total differential pressure along the pump is shown and compared with experimental results for the tested fluids at different pump rotations in Figure 14. As expected from previous studies [10][29], the water flow rate profile is not linear because there is a more significant presence of the pump's slippage phenomena. At zero differential pressure, the flow rate is maximum. Increasing the outlet pressure, the leakage occurs from the high-pressure cavity to the low-pressure cavity.

Due to the clearance between the rotor and the stator in metallic PCP, the metallic PCP gives higher leakage. As mentioned in section 0 , there is a regime of turbulence with $200700<\operatorname{Re}<41000$. Therefore, in the regions near the clearance, the velocities reached are significantly higher due to their low viscosity compared with the oils.

Similarly, the oils used in this study have a laminar behavior so that their volumetric flow profile is linear. Considering this, the flow profile slope concerning pressure is a function of the fluid's viscosity, which is 
increased by $88 \%$ more for API 31.5 light crude, $50 \%$ for API 22 intermediate crude, concerning API 12.8 heavy crude at $100 \mathrm{rpm}$. Therefore, the Overset mesh can characterize the fluid's behavior in different regimes by adding the turbulence model without requiring additional refinement.
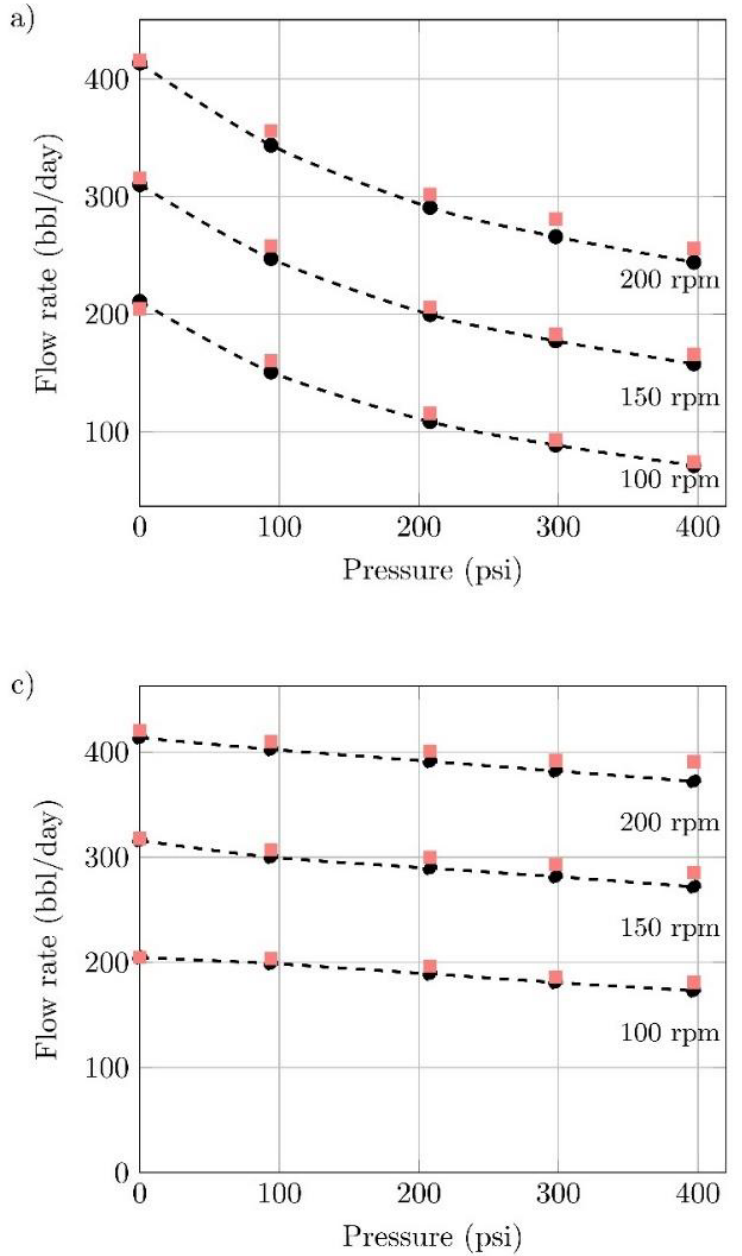
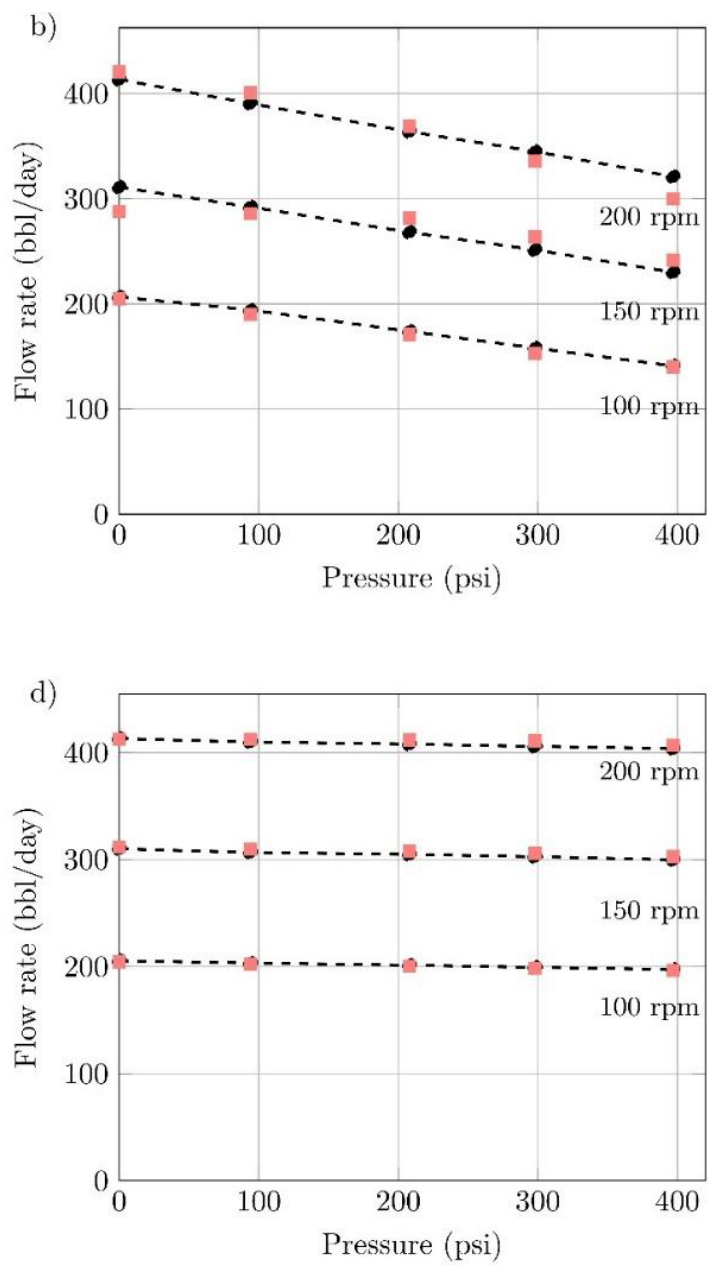

\section{- CFD = Experimental}

Figure 14. Experimental and CFD results of volumetric flow rate versus differential pressure at different pump rotations for a) water, b) Oil API 31.5, c) Oil API22.6, and d) Oil API 12.8

A similar analysis can be carried out from Figure 15 for the brake and hydraulic power of the PCP. It is possible to show that the pump's brake power increases with the fluid's viscosity increase since more energy is required. API 12.8 crude oil at $200 \mathrm{rpm}$ and $400 \mathrm{psi}$ involves a power of $15 \mathrm{HP}$, while for API 22.6 crude oil, there is a $20 \%(\sim-5 \mathrm{HP})$ reduction in the torque, for API 31.5 oil of $43 \%$ ( - $6.5 \mathrm{HP})$ and water of $67 \%(\sim-10 \mathrm{HP})$. Similarly, for all rotation speeds, this torque degradation is the same for all pressure points evaluated despite increasing shear stress. Nevertheless, an RMSE of about $10 \%$ is obtained because the friction losses associated with the rotor-stator interaction caused by the increase in the pressure difference in the stator material were not modeled. On the other hand, regarding the hydraulic power consumed by the machine for the three different speeds, it is possible to evidence that it increases as the rotation speed and the viscosity of the fluid increases. There is greater accuracy in the prediction of this variable through CFD. with an RMSE below 5\%. 

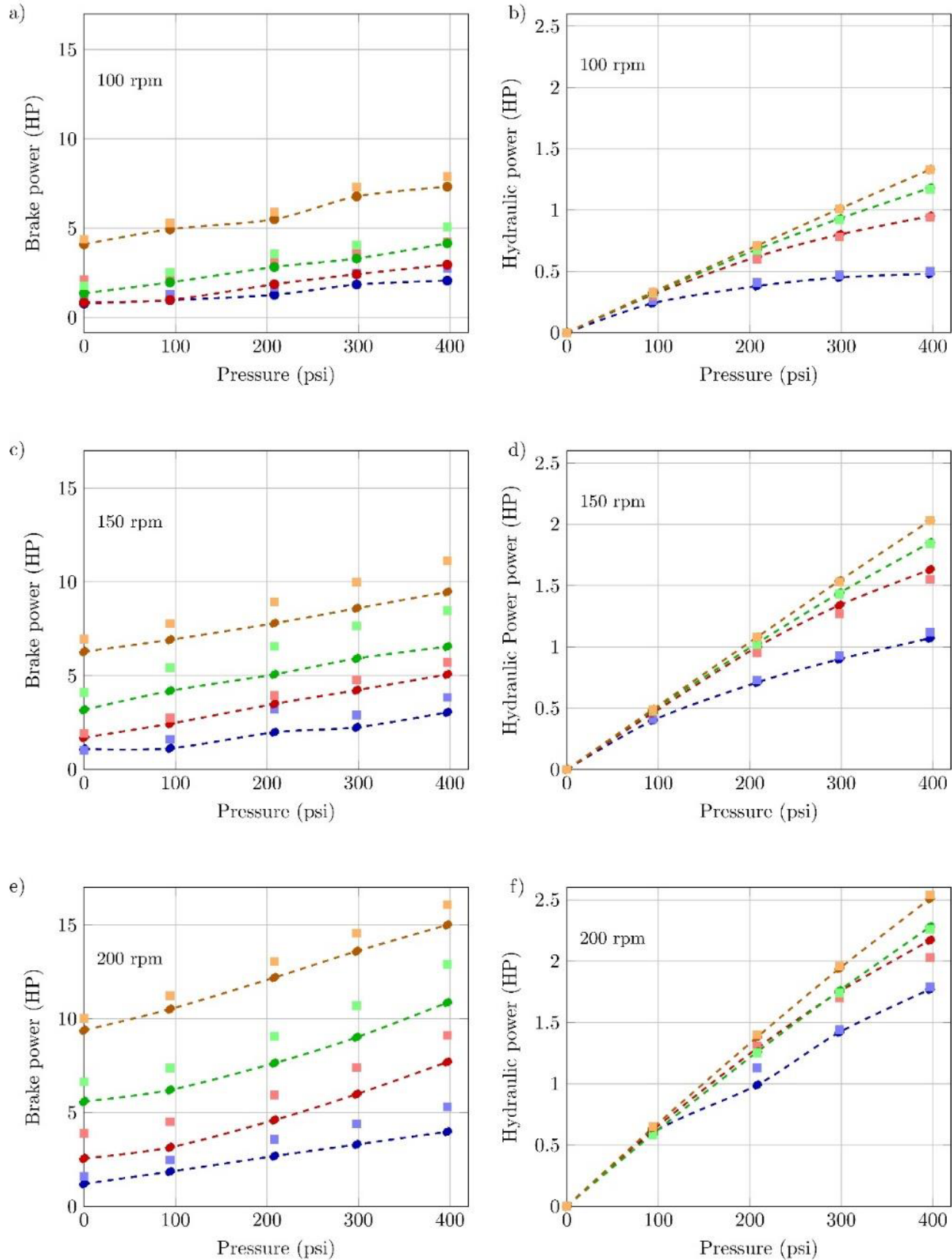

\begin{tabular}{lccccc}
$-\bullet$ & CFD Water & Experimental Water & $-\bullet$ & CFD API 31.5 & Experimental API 31.5 \\
- & CFD API 22.6 & Expcrimental API 22.6 & - & CFD API 12.8 & Expcrimcntal API 12.8 \\
\hline
\end{tabular}

Figure 15. Experimental and CFD results of brake and hydraulic power versus differential pressure at different pump rotations.

\section{Conclusions}


It is possible to see that through this new meshing technique, it is possible to represent the behavior of a progressive cavity pump with errors of less than $10 \%$ for all the operational variables studied. Likewise, it is possible to capture through this mesh the turbulence effects associated with water modeling without problems since the flow values have errors below $5 \%$. A simplified analysis was also presented, which provide some qualitative information about the impact of the main PCP geometrical parameters and fluid properties on pump performance. Numerical results are in concordance with expected trends, pointed by this analysis. The computational model developed can become a handy tool for PCP design and operation optimization and control.

\section{References}

[1] R. Moineau, “A New Capsulism," University of Paris, 1930.

[2] J. Chen, H. Liu, F. Wang, G. Shi, G. Cao, and $\mathrm{H}$. Wu, "Journal of Petroleum Science and Engineering Numerical prediction on volumetric ef fi ciency of progressive cavity pump with fl uid - solid interaction model," $J$. Pet. Sci. Eng., vol. 109, pp. 12-17, 2013, doi: 10.1016/j.petrol.2013.08.019.

[3] B. Wu and X. Li, "The special successful PCP applications in heavy oilfield," Soc. Pet. Eng. Progress. Cavity Pumps Conf. 2010, no. September, pp. 51-60, 2010, doi: 10.2118/136817-ms.

[4] G. Vetter and W. Wolfgang, "Understand Progressing Cavity Pumps Characteristics and Avoid Abrasive Wear." p. 14, 1995.

[5] A.-S. Elisse, "Analysis and Prediction of Fluid Flow Behavior in Progressing Cavity Pumps," vol. 139, no. December 2017, pp. 1-11, 2018, doi: $10.1115 / 1.4037057$.

[6] S. Noonan, Progressing cavity pumps, vol. 356. 1996.

[7] C. Wittrisch, PROGRESSING CAVITY PUMPS. 2013.

[8] I. R. Belcher, "An investigation into the operating characteristics of the progressive cavity pump," Cranfield University, 1991.

[9] A. Olivet, P. Epm, J. Gamboa, F. Kenyery, and U. S. Bolívar, "SPE 77730 Experimental Study of Two-Phase Pumping in a Progressive Cavity Pump Metal to Metal," 2002.

[10] J. Gamboa, J. Iglesias, and P. Gonzalez, "Understanding the Performance of a Progressive Cavity Pump with a Metallic Stator," 2002.

[11] S. F. A. Andrade, J. V Valério, and M. S. Carvalho, "Asymptotic Model of the 3D Flow in a Progressive Cavity Pump."

[12] E. E. Paladino, J. A. Lima, P. A. S. Pessoa, and R. F. C. Almeida, "Journal of Petroleum Science and Engineering A computational model for the fl ow within rigid stator progressing cavity pumps," J. Pet. Sci. Eng., vol. 78, no. 1, pp. 178192, 2011, doi: 10.1016/j.petrol.2011.05.008.

[13] J. A. De Lima, "A SIMPLIFIED MODEL FOR THE FLOW IN A PROGRESSIVE CAVITY PUMP," no. 2003, 2009.

[14] P. A. S. Pessoa, "Simulação Computacional do Escoamento em Bombas de Cavidades Progressivas," Universidade Federal do Rio Grande do Norte, 2009.

[15] J. Chen, H. Liu, F. Wang, G. Shi, G. Cao, and $\mathrm{H}$. Wu, "Numerical prediction on volumetric efficiency of progressive cavity pump with fluid-solid interaction model," J. Pet. Sci. Eng., vol. 109, pp. 12-17, 2013, doi: 10.1016/j.petrol.2013.08.019.

[16] T. Nguyen, E. Al-safran, A. Saasen, and O. Nes, "Journal of Petroleum Science and Engineering Modeling the design and performance of progressing cavity pump using 3-D vector approach," J. Pet. Sci. Eng., vol. 122, pp. 180186, 2014, doi: 10.1016/j.petrol.2014.07.009.

[17] T. Nguyen, H. Tu, E. Al-safran, and A. Saasen, "Journal of Petroleum Science and Engineering Simulation of single-phase liquid $\mathrm{fl}$ ow in Progressing Cavity Pump," 2016, doi: 10.1016/j.petrol.2016.09.037.

[18] K. R. Mrinal and A. Samad, "CFD SIMULATION OF FLOW THROUGH PROGRESSIVE CAVITY PUMP."

[19] E. Al-Safran, A. Aql, and T. Nguyen, "Analysis and Prediction of Fluid Flow Behavior in Progressing Cavity Pumps," J. Fluids Eng. Trans. ASME, vol. 139, no. 12, pp. 1-11, 2017, doi: $10.1115 / 1.4037057$.

[20] J. P. Valdés et al., "Comparative analysis of an electrical submersible pump's performance handling viscous Newtonian and nonNewtonian fluids through experimental and CFD approaches," J. Pet. Sci. Eng., vol. 187, 2020, doi: 10.1016/j.petrol.2019.106749.

[21] J. P. Valdes, "Experimental and Numerical Analysis of an Electrical Submersible Pump's Performance Handling Non-Newtonian Complex Flows," Universidad de los Andes, 2019.

[22] J. Gamboa, A. Olivet, G. D. S. C. A, S. Espin, and U. S. Bolívar, "SPE 84137 New Approach for Modeling Progressive Cavity Pumps Performance," 2003.

[23] P. Laws, J. S. Saini, and A. Kumar, "A Study on OpenFOAM's Overset Mesh Support Using Flow Past NACA 0018 Airfoil," no. July, pp. 118, 2019, doi: 10.20944/preprints201907.0217.v1.

[24] X. Qiu and M. R. Anderson, "Analysis and Validation of a Unified Slip Factor Model for Impellers at Design and," vol. 133, no. October, pp. 1-9, 2011, doi: 10.1115/1.4003022.

[25] M. Peric and S. Ferguson, "The advantage of polyhedral meshes," Dynamics, vol. 24, p. 45, 
2005.

[26] J. P. Valdes, D. Becerra, D. Rozo, A. Cediel, F. Torres, and M. Asuaje, "Comparative analysis of an electrical submersible Pump's performance handling viscous Newtonian and non-Newtonian fluids through experimental and CFD approaches," J. Pet. Sci. Eng., no. xxxx, 2019.

[27] J. H. Ferziger, M. Peric, and A. Leonard, "Computational Methods for Fluid Dynamics," Phys. Today, vol. 50, no. 3, pp. 80-84, 1997, doi: $10.1063 / 1.881751$.

[28] B. Andersson, R. Andersson, L. Hakansson, M. Mortensen, and B. G. M. van Wachem, Computational fluid dynamics for engineers. 2012.

[29] E. E. Paladino, J. A. Lima, R. F. C. Almeida, and U. Dem, "SPE 114110 Computational Modeling of the Three-Dimensional Flow in a Metallic Stator Progressing Cavity Pump," no. 2003, 2008. 